\title{
A obesidade infantil no Brasil e fatores associados: desafios para os professores de educação física.
}

The childish obesity in Brazil and associated factors: challenge for Physical Education teachers.

\author{
Emanuel Nogueira \\ Faculdade Norte do Paraná-UNOPAR (Brasil) \\ vigilantepb@hotmail.com \\ Francisco de Assis do Nascimento \\ Faculdade Norte do Paraná-UNOPAR (Brasil) \\ assisbmrcc@hotmail.com \\ Renan Lima de Souza \\ Faculdade Norte do Paraná-UNOPAR (Brasil) \\ renanlima-boy@hotmail.com \\ Wanderson Marcelino da Silva \\ Faculdade Norte do Paraná-UNOPAR (Brasil) \\ wandersonmarcelino19@hotmail.com
}

Fecha recepción: 01-11-2019

Páginas: $13-24$

Fecha aceptación: 25-12-2019

\section{Resumo.}

A obesidade infantil em nossa sociedade tem se desenvolvido de forma significativa e assustadora. Caracterizando um problema de saúde pública que envolve vários aspectos relevantes, decorrente de sua manifestação lenta e tranquila tornando-se muito perigosa, além de desencadear outras doenças metabólicas e cardiovasculares. Nesse contexto, esse artigo prima em realizar uma pesquisa bibliográfica como objetivo de atualizar novas pesquisas na área em discussão, bem como criar oportunidade de reflexão dos docentes de Educação Física no processo de conscientização dos alunos sobre a importância da atividade física no combate à obesidade. Em se tratando de uma pesquisa bibliográfica aqui foram abordados alguns referentes pesquisadores dessa temática. Por fim, conclui-se que esse tema é de grande magnitude no atual contexto educacional e de saúde pública, sendo possível o processo de conscientização pelos professores de Educação Física e demais professores de outra disciplina através de um trabalho interdisciplinar.

Palavras-chave: educação física escolar; ensino-aprendizagem; obesidade; atividade física; conscientização

\section{Abstract.}

The childish obesity in our society has increased in a significant way. Portrayed a Public health problem that involves lots of relevant aspects, manifest it slowly and 
safe journey become very dangerous, besides develops others metabolic and cardiovascular diseases. In this context, this article realized a Bibliography research with the objectives of update news researches about this theme; besides create a great opportunity for the Physical Education teachers think about the awareness with the students and the Physical activities battle with the obesity. This is a bibliography investigation and here it can see excellent researchers about this theme. Finally, it concludes that the theme is very important nowadays in many contexts of education or public health, it's been possible a great awareness process through the Physical Education Teachers and others teachers with cross-curricular work.

Key words: physical education; obesity; learning teach; physical activities; awareness

\section{1.-Introdução.}

Nesse trabalho de pesquisa será abordado o tema sobre a Obesidade infantil no Brasil e fatores associados, pois esse tema é considerado na atualidade um problema e um grande desafio de saúde pública mundial.

A Obesidade é considerada uma doença crônica, pois se encaixa naquelas doenças que se manifestam de forma lenta e com duração longa. Ela é causa de milhões de mortes no mundo inteiro, tendo como consequência outras doenças, como: diabetes, parada cardíaca, câncer, doenças respiratórias e doenças cardiovasculares. Chega de forma lenta e não respeita cor, raça, crença, escolaridade, aquisição econômica e está presente em diversos países independente do desenvolvimento social ou econômico desses países.

Assim, muitos estudos são desenvolvidos por órgãos competentes no intuito de prevenir, amenizar e conscientizar as pessoas sobre esse grave problema. A Organização Pan-Americana da Saúde (2003) desenvolve pesquisas dentro dessa temática no intuito de alertar a população mundial e gestionar programas de prevenção visando uma melhoria na qualidade de vida da população mundial.

A Educação Física escolar é uma interessante oportunidade para despertar e esclarecer novos conhecimentos aos educandos e seus familiares a respeito de hábitos alimentares mais saudáveis e no estilo de vida adequado e incentivo a prática esportiva que priorize atividade física.

Deste modo, esse trabalho objetiva-se contribuir para que o discente obtenha conhecimentos substanciais sobre Epidemiologia em Educação Física e fatores determinantes para a saúde, e sobre 0 aumento da prevalência da obesidade infantil. Refletir sobre os desafios da área da Educação Física e sua atuação prática à prevalência da obesidade infantil, em ambiente escolar e não escolar. Compreender a importância da interdisciplinaridade de conhecimentos e sua contribuição na tentativa de diminuir a obesidade infantil em ambiente escolar e não escolar. A metodologia empregada trata-se de pesquisa bibliográfica descritiva, pois é 0 
resultado de pesquisa minuciosa através de artigos na base do Google Acadêmico sobre a temática referente às palavras-chaves: Educação física escolar, ensinoaprendizagem, obesidade, atividade física, conscientização.

\section{2.-Desenvolvimento.}

O problema da Obesidade infantil no Brasil tem levado a inúmeras consequências na saúde das pessoas, caracterizando dessa forma um problema de saúde pública. Daí a necessidade de compreender o papel da Educação Física na solução ou prevenção dessa doença através dos vários processos a mencionar o equilíbrio do consumo alimentar e prática de exercícios físicos.

Importante ressaltar a aptidão física e motora em crianças e adolescentes. Ao falarmos em "aptidão física", devemos levar em consideração sua definição e importância na contribuição da saúde de crianças e adolescentes. Assim, é preciso definir esse termo: "Aptidão física é a capacidade de cada um de nós realizarmos as tarefas do dia a dia e pode ser melhorada através do exercício físico." (Vasconcelos, 2019). O mais relevante é saber sua importância no processo de uma boa qualidade de vida das pessoas e em especial nessa pesquisa, nosso foco: crianças e adolescentes.

De acordo com Pereira e Moreira (2013), o conhecimento dessas aptidões de crianças e adolescentes é importante no tocante de especificar e atualizar 0 conhecimento, para poder gerar futuros benefícios a essa população, valendo ressaltar que esses cuidados necessários terão uma boa repercussão na vida futura de cada indivíduo, transformando assim, em uma boa qualidade na saúde desses indivíduos.

Dessa forma, surge o profissional da Educação Física para promover a aptidão física desse público mencionado, partindo do pressuposto que o exercício e a atividade física oferecem inúmeras possibilidades para aumentar a condição de uma boa qualidade de vida, em especial a uma boa saúde. Por isso, Pereira e Moreira (2013), mencionam que a Educação Física como ciência autônoma, tendo em seu próprio objeto de estudo (movimentos, motricidade humana, ação e movimento humano), argumentos para disseminar a importância do papel do educador, bem como informar, acompanhar, direcionar exercícios para uma futura avaliação desse processo.

A Obesidade e Sobrepeso assim, definindo o conceito de Obesidade e sobrepeso, Editorial Conceito (2016) enfatiza que a obesidade se trata de um transtorno que se caracteriza pelo aumento excessivo da proporção de gordura corporal podendo colocar a saúde em risco. É ressaltado também, que a obesidade está relacionada a outros distúrbios, a exemplo de problemas respiratórios, circulatórios, e outros relacionados a hábitos alimentares e de exercício físicos, que recentemente está mais associado não somente aos idosos, mas também às crianças e adolescentes. 
Por isso, é importante lembrar que a obesidade se apresenta de vários níveis, porém é a mórbida a mais severa para a vida da pessoa que é acometida. Nesse caso, é preciso se submeter a um exame específico denominado de índice de massa corporal. Nesse exame é possível associar o peso à altura da pessoa, a princípio analisando que quanto maior a altura, maior o peso. Os resultados são classificados através de vários níveis: baixo peso, normal, sobrepeso ou obesidade mórbida. (Editorial Conceito, 2016).

Segundo Guimarães (2017), a população do nosso planeta está ganhando peso de maneira muito rápida, com uma incidência muito grande entre crianças e adolescentes. Essa é uma realidade de vários países, segundo essa pesquisadora na revista científica The Lancet, a taxa de obesidade em crianças disparou em 41 anos. Essa realidade preocupa todos nesse momento porque futuramente esses dados alarmantes, vão a se transformar em problema de saúde pública, onde 0 alto índice de doenças associadas à obesidade, dentre elas: doença do fígado, diabetes e pressão arterial elevada.

Fatores que acusam a obesidade ainda de acordo com Guimarães (2017), a elevação dos níveis de obesidade no Brasil é semelhante à de outros países que estão sempre relacionados ao consumo de produtos industrializados, que tem uma grande quantidade de gordura e açúcar. 0 consumo em abundância está associado a alguns fatores, dentre eles: o preço mais baixo que o de alimentos saudáveis, e questão de status, já que a grande maioria da população mundial aprecia, tornam-se moda entre as pessoas, por se tratar de produtos saborosos e de fácil aquisição, pois a correria da vida cotidiana sugere uma alimentação mais rápida, barata e acessível.

Por isso, a Federação Mundial de Obesidade adverte aos países que o corrente risco de obesidade mundial, põe em risco a saúde pública, porque vai gerar problemas de saúde na população mundial. Para essa Federação a estimativa é que, em 2025, cerca de 150 mil crianças e jovens no nosso país desenvolverão diabetes, enquanto que 1(um) milhão terão pressão arterial elevada, além de outros problemas que surgirão, como: gordura no fígado.

Para Guimarães (2017) reforça ainda que muitas vezes as pessoas não querem aceitar que estão obesos, ou apenas são indiferentes a esses aspectos. Por fim, alguns cuidados são importantes na prevenção dessa doença, a mencionar: aumentar 0 aleitamento materno na infância, diminuir consideravelmente o consumo de alimentos gordurosos e rico em açúcar, onde os principais vilões continuam sendo 0 refrigerante, os biscoitos e a comida dos fast food. Não podemos esquecer a prática de exercícios físicos, mudar conceitos e hábitos.

\section{1-Disfunções crônico-degenerativas resultantes da obesidade.}

As doenças crônicas representam a principal causa de mortalidade e incapacidade na atualidade, assim entram nesse contexto: obesidade, diabetes, doenças cardiovasculares, câncer, doenças respiratórias. São chamadas de agravo não 
transmissíveis, pois não são transmitidas de pessoa a pessoa, conforme apontam os estudos da Organização Pan-Americana da Saúde (2003).

Para essa organização, hoje essas doenças atingem vários países, seja ele desenvolvido ou não, pois se tornou um problema mundial. Todo esse acontecimento vem do reflexo da nossa sociedade contemporânea que mudou completamente seu hábito alimentar, seu estilo de vida e o baixo desempenho de atividade física. Esse é o modelo de uma sociedade industrializada, fruto do desenvolvimento industrial, urbano e econômico.

Esse problema vem representar em números para a organização mundial de saúde cerca de milhões de pessoas que morrem de infarto e derrames. Assim, a Organização Pan-Americana da Saúde (2003).

Atualmente, os infartos e derrames matam 12 milhões de pessoas (7,2 por causa da cardiopatia isquêmica e 5,5 milhões devido a acidentes vasculares cerebrais). Além disso, 3,9 milhões de pessoas morrem por hipertensão e outras cardiopatias anualmente. (Organização Pan-americana da saúde, 2003, p. 8)

Assim, o mesmo estudo comprova que à mediada que aumenta a renda da população e ela torna-se mais urbana a alimentação é rica em carboidratos complexos ricos em gorduras saturadas e açúcares. Além do mercado de trabalho que exige menos esforço físico, uso de transportes automatizados, a tecnologia que facilita a vida e determinados trabalhos do dia a dia, ficando de lado as velhas e tradicionais atividades físicas de simples tarefa diária. (Organização Pan-americana da saúde, 2003).

2.2.-Áreas da Educação Física que podem contribuir na redução da prevalência da obesidade infantil no Brasil.

A Organização Pan-Americana (2003) menciona que é preciso gerenciar o controle de peso nas pessoas em situação de risco, com estratégias que vão desde a prevenção, manutenção do peso ou dieta, para perdê-lo.

Para essas estratégias é preciso um regime alimentar disciplinar e a conscientização da importância de exercícios físicos regulares, dessa forma a Educação Física vem em auxílio daqueles que necessitam dessa orientação, passando a frequentemente a engajar a atividade física em sua vida diária, como: caminhada, corrida, dança, prática de esportes, etc. Outra sugestão seria a ingestão de frutas, suco, verduras, vegetais, comida natural na dieta, alimentos ricos em fibras, além de eliminando as gorduras e açúcares, grandes causadores dessas doenças.

Esses cuidados que o profissional da educação física deve estar preparado para atuar em diversos ambientes seja ele escolar ou não.

\section{3.-A Educação Física escolar e o processo de prevenção da obesidade.}

A Educação Física Escolar é considerada muito importante na vida escolar das crianças e adolescentes, ela proporcionar mais saúde para seus praticantes, daí vem à importância dos professores de Educação Física incentivar os alunos no processo de conscientização dessas práticas. 
Já mencionado por Câmara (2010) na escola encontra-se um bom lugar para os discentes viverem suas experiências com a educação corporal. Com ela 0 aluno inicia seu processo psicomotor passando a desenvolver a corrida, o pular, o saltar e demais atividades psicomotoras, tão fundamentais para criança, adolescentes e adultos.

Para Câmara (2016) o papel dos jogos e brincadeiras é fundamental no desenvolvimento de uma criança, além de iniciar um processo de valorização e prevenção de enfermidades, a mencionar a obesidade, aprendem de forma positiva e passam a mudar seus hábitos alimentares seguidos de prática de atividade física, por isso a informação sobre o real problema é importante no processo de conscientização.

Assim, a influência dos professores de Educação Física nas escolas pode fazer um diferencial nas mudanças de atitudes alimentares e de comportamento com relação ao cuidado corpo e da saúde pelos alunos.

De acordo com, Araújo; Brito; Silva (2010) é realidade nas escolas brasileiras que os alunos da Educação Física escolar realizem movimentos não sistematizados, pouca prática de atividade física, poucos movimentos, e isto implica em um distanciamento entre teoria e proposições para as aulas.

Dessa forma, Matsudo e Matsudo (2006) alerta que o combate ao sedentarismo, passa a ser indispensável nos dias atuais. O sedentarismo é um dos fatores responsáveis pelo aumento da obesidade, pois a prática de atividade física regular promove algumas doenças e essa sem dúvida é a que tem aumentado significativamente os números de casos.

Para contribuir de maneira satisfatória com a saúde dos alunos, é preciso considerar que a prática de exercícios, desportos e aptidão física trata-se de um processo do indivíduo com experiências educacionais, por meio das práticas físicas e devem permanecer ao longo da vida. Uma boa combinação é a prática regular de exercícios físicos e alimentação saudável. (Araújo; Brito; Silva, 2010)

Dentro dessa perspectiva, Farias; Lara; Azevedo (2018) lembra que a Educação Física é um componente curricular referenciado pela Lei n. 9.394/1996 (LDB) e que está presente nas escolas. Esta disciplina objetiva contribuir na formação das crianças e jovens de forma interdisciplinar, através do trabalho dinâmico e do bom relacionamento com outras disciplinas.

Essa lei torna legítima a Educação Física na educação infantil e no Ensino Fundamental, porém não precisa de um professor qualificado para ministrar essa disciplina nessa fase escolar, esta abertura permite que apareçam alguns problemas, pois é visível a lacuna entre a teoria e a realidade, é compreensível que o professor dessa disciplina deva adquirir conhecimentos variados, amplos e críticos. (Farias; Lara; Azevedo, 2018). 
Juntamente com os professores de Ciências ou biologia trabalhar os fatores biológicos e químicos da obesidade, incentivando a importância da prática esportiva, o zelo pela boa alimentação e o cuidado com a saúde física e mental.

Desse modo, os professores das demais disciplinas podem unir-se com o professor de Educação Física e criar outras situações de aprendizado, unindo a teoria e a prática em razão do bem comum, podendo variar o uso da metodologia mais viável. Na disciplina de Geografia, é possível acrescentar na discussão os aspectos sociais, culturais, econômicos, políticos e geográficos sobre a temática fomentando novos conhecimentos na ajuda da reflexão e conscientização do problema.

Na disciplina de Primeiros Socorros, de acordo com Ribeiro (2019) toda a teoria sobre obesidade, poderá ser posta em prática toda vez que um indivíduo necessite de um procedimento de reanimação, diante de uma parada Cardiorrespiratória, queimadura, fratura, corte, um mal súbito devido às altas taxas de gorduras.

\section{4.-Saúde na escola, questão de educação e direito.}

O direito a saúde é algo conquistado mundialmente ele foi assegurado pela Organização das Nações Unidas (ONU) em 1948, juntamente com a Declaração Universal dos Direitos Humanos. Assim, Bressan (2008) afirma que antes dessa data, a saúde era um privilégio para uma parte da população que podia contribuir com a previdência social, enquanto que aos trabalhadores a assistência à saúde dependeria do vínculo empregatício. Em suma, a maior parte da população brasileira não tinha acesso à saúde conforme deveriam ter.

Porém, na década de 60 com a realização da $8^{a}$ Conferência Nacional de Saúde em 1986, que defendia a bandeira deque a saúde é um direito de todos e também um dever do estado, foi uma grande conquista para as futuras gerações diante do tema saúde e educação. Esse ideal ganhou força e em 1988 com a promulgação da Constituição Federal desse ano, a saúde passou a fazer parte do Sistema de Seguridade Social, sendo instituído o Sistema Único de Saúde (SUS). Com essa novidade, a saúde passou a ser vista com mais atenção e cuidados, passando a integrar muitas ações do governo federal visando à vigilância, promoção e restabelecimento da saúde brasileira. (Bressan, 2008)

Desde então, o mesmo autor afirma que o SUS baseia-se em princípios éticopolíticos, como: a universalidade do acesso, a integridade da atenção, a equidade $e$ a participação social. A universalidade do acesso se caracteriza pelo direito do acesso da população à saúde de todos os níveis, sem preconceitos ou outro fator que dificulte esse acesso. A integridade de atenção trata-se de ações e serviços de prevenção, cura em todos os níveis de complexidade de saúde, seja individual ou coletivo. A equidade fundamentada na igualdade e implementação de estratégias para recuperação da saúde de pessoas. A participação social firma o direito das pessoas da aquisição da gestão do SUS, por meio de conselhos de saúde, gestão 
participativa no monitoramento avaliando as políticas públicas voltadas à saúde da população.

Assim, com a fundação do SUS em um sistema repleto de cuidado à saúde melhorou em muito a vida da população brasileira, porém não chega a atingir todos, pois as evidências apontam as dificuldades, limites e diversos fatores articulados ao Estado através de sua ação sistêmica e intersetorial, primando pela relevância do social, econômica e cultural.

Para Bressan (2008) foi em 1953 o ministério da Educação fracionou em dois ministérios: Ministério da Educação e Cultura e Ministério da Saúde, passando os dois a terem autonomia e responsabilidades distintas, passando a ter autonomia para a elaboração de suas políticas. Conforme visto, esses dois ministérios têm muito em comum desde sua origem até os dias atuais, além de muita semelhança em seu progresso histórico, mesmo assim tornaram-se distantes em seus ideais.

Por meio de articulações entre Ministério da Saúde e da Educação, respeitando seus saberes e práticas, começam uma articulação no sentido de propor ações de saúde na escola. O objetivo principal é a melhoria da qualidade de vida na busca de soluções de problemas, nela os autores envolvidos nesses dois espaços devem propor atividades que façam sentido a ambos. (Bressan, 2008)

Os desafios da contemporaneidade remetem os diversos setores a uma reflexão, análise em busca de alternativas cada vez mais sólidas na redução de problemas, como: contaminação, violência, questões ambientais, doenças transmitidas por vírus bactérias, fungos. Por isso, através da Política Nacional de Promoção da Saúde (PNPS) em 2006 firmou-se a importância do diálogo entre setores da sociedade com a participação de diversos setores. (Bressan, 2008)

Nesse contexto foram articuladas muitas políticas de saúde passando a unir a educação e saúde, no intuito de articular ações com a participação da comunidade, escola e unidade básica de saúde. Priorizando as parcerias no trabalho de promover saúde, prevenção e apoio às práticas de conscientização de ações alimentares, atividades físicas, práticas corporais e ambientes saudáveis. Dessa forma, 0 ambiente escolar apresenta um contexto favorável, para essas novas ações culminando com a interação entre saúde e educação. No que se refere às práticas observam as diversas realidades e os diversos sujeitos, planejando e realizando partilhas, saberes, histórias de forma individual ou coletiva, capaz de resolver e realizar as melhoras. (Bressan, 2008).

Assim, Silva (2008) reafirma que de acordo com a LDB/96 (Lei de Diretrizes e Base da Educação Básica) a educação é um direito da família, sendo obrigação do estado à participação dos profissionais da educação respeitando alguns princípios de liberdade e solidariedade. Sendo assim, a partir do século XX os debates de saúde na rede escolar, ficou mais intenso com o surgimento de especialistas em torno da saúde escolar, intensificado por algumas situações relacionadas à sexualidade, métodos contraceptivos, gravidez na adolescência, DST (Doenças Sexualmente 
Transmitidas), violência, drogas, prevenção de acidentes, saúde oftalmológica, odontológica, psicológica, preservação do ambiente saudável, e, sobretudo alimentação.

Uma forma interessante seria a integração do tema saúde na escola ser repensada sobre o ponto de vista de uma conexão com o PPP (Projeto Político Pedagógico) da escola, pois até pela organização desse instrumento quando envolve todos integrantes do ambiente escolar se consolida em verdadeira ação para minimizar as problemáticas abordadas. (SILVA, 2008)

De acordo com Silva (2008), vale ressaltar que as instituições de saúde e educação precisam desempenhar um papel de protagonista ao abrir espaços, criar situações favoráveis aos educandos no contexto escolar, essa decisão permite uma aproximação mais eficaz entre, escola, família e saúde. Não dá para separar ou dissociar a promoção da saúde na escola, ela favorece o conhecimento por meio de políticas sociais, que objetivam diminuir o preconceito, as dificuldades, questões sociais de cor, raça, orientação sexual e gênero.

O professor é um condutor de todo esse processo, assim na prática educativa, o ponto de vista do professor, sua prática e conhecimento prévio e conhecimento adquirido deve fazer todo o diferencial. 0 aluno nesse processo também tem um papel primordial, porém sempre compreendendo suas significações, diante de uma metodologia participativa. O professor conseguirá alcançar bons resultados através de planejamento na construção de atividades, programas, projetos que venham a valorizar a vida de jovens e adolescentes. Silva (2008) acredita que criando ambientes favoráveis à saúde, autoestima, valorizar o outro, oportunizar qualidade de vida, ambientes favoráveis, estimular a alimentação saudável, práticas de atividades físicas, valorizando o outro na busca de parcerias entre nas mais diversas áreas da saúde: ocular, auditiva, mental, bucal sempre em união com os agentes escolares.

Assim, a atividade física mesmo sendo um direito se apresenta com várias dificuldades nas escolas, aqui se pode mencionar aspecto físico, pois as escolas não dispõem de um bom ambiente físico, falta de uma aula bem planejada, ausência muitas vezes do professor de educação física, falta de incentivo aos alunos pela prática esportiva, não há incentivo aos menos favoráveis ao esporte, etc. são algumas situações que geram muitos problemas na evolução da Educação Física. (Farias; Lara; Azevedo, 2018)

Dessa forma, o mesmo autor reforça a importância da atividade física e a integração nos programas formais sempre objetivando as condições favoráveis para as crianças e adolescentes. Muitas são as melhorias sugeridas para as aulas de Educação Física, a começar pelo interesse dos alunos, através da disposição dos professores no manejo de suas metodologias, planejamento e participação no incentivo dos alunos na prática esportiva. Incrementar as atividades com: dança, ideias inovadoras, incentivo a natação, atletismo, corridas, etc. (Farias; Lara; Azevedo, 2018) 


\section{5.-Prática de atividade física nas escolares para alunos sobrepeso.}

A Disciplina de Educação Física na escola e o professor dessa disciplina assumem um papel fundamental na prevenção e no tratamento de obesos além dos predispostos a obesidade, para esse feito é preciso oferecer $m$ ambiente propício aos alunos e acessível a práticas esportivas, aumentando os níveis de atividades relacionadas ao esporte, diariamente para as crianças, jovens e adolescentes. Associar temas de saúde e obesidade e relacioná-los a atitudes e mudanças de hábitos alimentares, atividade física e reformulação de valores. (Costa et al, 2001).

0 excesso de peso influencia negativamente na vida de pessoas acometidas pelo sobrepeso. De forma geral, as pessoas sofrem vários preconceitos, não conseguem desenvolver boa atividade física, como: corridas, deslocamento, agilidade, essa forma negativa gera um desânimo naqueles que não conseguem acompanhar os demais que mantem seu desenvolvimento corporal normal. Infelizmente as pesquisas configuram que ocorre em ambos os sexos, hoje meninos e meninas são acometidos do sobrepeso.

Por isso, uma boa opção é a prática de esportes sistematizada no ambiente escolar, porém sabe muito bem que a maioria das escolas não oferecem essa oportunidade nem um ambiente acolhedor e propicio às modalidades esportivas. Muitos professores não estão preparados para desenvolver atividades na modalidade de atividade física e desenvolvimento esportivo.

\section{3.-Conclusão.}

Ao final desse trabalho percebe-se a grande importância do conhecimento científico na vida das pessoas, pois é preciso ter conhecimento para poder mudar situações melhorando assim a vida das pessoas em diversos setores, aqui em especial na saúde de crianças, adolescentes e adultos, acometidos pela Obesidade que atualmente vem ceifando vidas de pessoas tão jovens e produtivas.

Através dessa pesquisa percebe-se o papel do professor de Educação Física e suas muitas responsabilidades, dentre elas a de educar e incentivar os alunos na prática esportiva, mostrando para eles a importância de permanecer ativo desde a infância, mas é preciso também uma preparação do educando sobre a temática da obesidade e seus efeitos na sociedade e na vida dos alunos, porém para um melhor resultado é preciso repensar a carga horária de Educação Física nas escolas, pois atualmente apresenta uma carga horária insuficiente para desenvolver um trabalho específico com os alunos.

Essa foi uma grande oportunidade para refletir sobre os desafios da área da educação física na prevenção da obesidade infantil, em ambientes escolares ou não, pois se percebe que este estudo é pertinente à comunidade acadêmica além da sociedade em geral. Compreendemos que o professor de Educação Física tem um grandioso papel no processo de conscientização de uma vida mais saudável dos alunos, alimentação e prática esportiva. 
Essa falta de sistematização prejudica o trabalho dos professores de Educação Física, pois estes têm que apresentar um maior desejo de efetivar essas mudanças, pois muitas vezes eles encontram uma rejeição dos próprios colegas que não tem 0 mesmo ânimo para a mudança e incentivo.

Dessa forma, os objetivos específicos foram alcançados à medida que contribuiu de forma firme para que os professores de Educação Física ampliem seus conhecimentos sobre o tema. Percebam que muitos são os desafios na área da Educação Física e sua atuação através do apoio de outros professores com projetos multidisciplinares.

Percebe-se que é muito importante um novo olhar sobre esta temática, e que ela venha a contribuir de forma firme e saudável, buscando cada vez mais novos conhecimentos e entendimento das problemáticas sociais que envolvam a saúde e a escola. Nesse foco, prevalece a importância por mais conhecimento, para entender e solucionar a problemática.

Enfim, percebemos que a interdisciplinaridade de conhecimentos é uma opção eficaz na tentativa de diminuir essa problemática, como também redefinir e modelar nas pessoas mais propensas à obesidade hábitos saudáveis. É preciso impulsionar um novo olhar sobre o tema obesidade, com esse ato é possível a colaboração de uma sociedade mais saudável, com pessoas mais satisfeitas.

\section{Referências.}

Araújo, R.A., Brito, A.A., Silva, F.M. (2010). O papel da educação física escolar diante da Epidemia da obesidade em crianças e adolescentes. Educação Física em Revista. ISSN: 1983-6643, vol. 4 nº 2 mai/jun/jul/ago.

Bressan, A. (2008). O que escola tem a ver com saúde? Saúde e educação: interfaces possíveis. Salto para o futuro. Ano XVIII boletim 12 - agosto- 2008. ISSN 1982 - 0283. Rio de janeiro - RJ.

Câmara, S.A.S. (2016). Psicomotricidade e Trabalho Corporal. São Paulo: Pearson Educação do Brasil.

Costa, E.Q., Ribeiro, V.M.B., Ribeiro, E.C.O. (2001). O Programa de alimentação escolar: espaço de aprendizagem e produção de conhecimento. Revista $\mathrm{Nu}$ Revista Nutrição, v. 14, n 13,p.225. São Paulo, 9 de setembro e dezembro 2001.

Farias, G., Lara, A.F.C., Azevedo, A.L.P.F. (2018). A obesidade em crianças e adolescentes versus disciplina de Educação Física na rede pública de ensino. Disponivel em:< http://dspace.unibrasil.com.br:8080/xmlui/handle/123456789/111>. Acessado em: 04/11/2019.

Guimarães, K. (2019). Brasil terá 11,3 milhões de crianças obesas em 2025, estima organização. BBC NEWS Blog., 11 out. 2017. Disponível em: 
<https://www.bbc.com/portuguese/geral-41588686>. Acessado em: 15 de maio de 2019.

Matsudo, V.K.R., Matsudo, S.M.M. (2006). Atividade física no tratamento da obesidade. Einstein. 4(supl.1):S29-S43.

Organização Pan-americana Da Saúde (2003). Doenças crônico-degenerativas e obesidade: estratégia mundial sobe alimentação saudável, atividade física e saúde. Brasília, 2003. Disponivel em: < www.luzimarteixeira.com.br/wp.../11/livro-saude-e-doencas-cronicodegenerativas.pdf $>$. Acessado em 15 de maio de 2019.

Pereira, E.S., Moreira, O.C. (2013). Importância da Aptidão física relacionada à saúde e aptidão motora em crianças e adolescentes. Revista Brasileira de Prescrição e Fisologia do Exercício, São Paulo, v.7, n.39, p.309 -316. Maio/Jun. 2013. ISNN 1981- 9900 versão eletrônica. Periódico do Instituto Brasileiro de Pesquisa e Ensino em Fisiologia do Exercício. Disponível em: <www.ibpefex.com.br.> Acessado em: 15 de maio de 2019.

Ribeiro, K.D.K.F. (2019). Primeiros Socorros. Brasil Escola. Disponível em: < https://brasilescola.uol.com.br/saude/primeiros-socorros.htm>. Acesso em 16 de maio de 2019.

Silva, C.S. (2008). O que a escola pode fazer para promover a saúde de crianças, adolescentes e jovens? Salto para o futuro. Ano XVIII boletim 12 - Agosto2008. ISSN 1982 - 0283. Rio de janeiro - RJ.

Vasconcelos, J.P. (2019). Educação Física. Disponível em: <https://pedroedfisica.webnode.pt/capacidades-fisicas>. Acesso em: $15 \mathrm{de}$ maio de 2019. 